\title{
BIMPO: A GENERATIVE PARAMETRIC TECHNIQUE FOR BUILDING ENVELOPE DESIGN
}

\author{
AIDA GAMAL FATHY, ZEYAD EL-SAYAD, DINA SAADALLAH \& ALI FOUAD BAKR \\ Department of Architecture, Faculty of Engineering, Alexandria University, Egypt
}

\begin{abstract}
Parametric design tools are digital tools used by the architect, for the purpose of creativity. BIM platforms tend to simplify the work of designers and engineers, if their products are within standards. Within the digital construction field, creativity and information modelling are frequently in contrast. An interaction between parametric design and BIM platforms is necessary to avoid the influence of predefined parametric elements on the design concept. Parallel origami is suggested as a generative tool to produce complex shapes. This research proposes a new tool for Revit architecture software (BIM tool), based on the BIM-Parametric-Origami (BimPO) integration framework. This tool directly generates optimized alternatives of complex or non-conventional solar devices, for specific target performances of building envelopes. The BimPO script provides more possibilities for an increasing number of form generation. Results will prove BimPO's ability to efficiently achieve numerous Origamic Solar Device (OSD) alternatives, for improved energy efficiency. Moreover, selection criteria are proposed, which could indicate optimal OSD configurations, with a balance of daylight and thermal performance (TH), based on daylight simulation.
\end{abstract}

Keywords: BIM, parametric design, programming, visual programming, origami, generative design, form generation, solar devices, building envelope.

\section{INTRODUCTION}

Building Information Modelling (BIM) is an intelligent 3D model-based process, which improves the output and management performance of projects. Adaptation of BIM is steadily gaining popularity in the construction industry from $13 \%$ in 2011 to $74 \%$ in 2018 . Users have been quite aware of BIM, but its adoption still varies from one country to the next (Denmark 74\%, Canada 67\%, UK 48\%, Japan 46\%, and the Czech Republic 25\%) [1]. Moreover, the usage of BIM is growing and within five years, it is predicted that BIM usage in the design professions in most countries will reach more than $80 \%$. These facts suggest that the use of BIM is turning into the norm within the construction field [2].

Improvements in the technology of building construction have accelerated within the latest few years and developments in BIM have been essential to fulfil the more recent requirements. with the rise in complexity that has come with contemporary architecture, whether it is to do with the complexity of form, the need for better performance, or facility management, there is a greater need for tools to help the process of form generation. As the number of digital tools in use increases, there is a greater need for interoperability between different applications. BIM tools allow the integration between various disciplines, by having a semantically rich model to-and-from which data is transferred [3].

These issues in the early phase of design are counteracted by the use of conceptual modelling tools, such as in Revit. These tools often create more general and simpler simulations, which yield an approximation of the outcome of the process. This includes the distribution of solar energy in a building throughout the year or an approximation of the most optimal window glazing. VPL (Visual Programming Language) software has been the most recent addition to modelling tools. VPL is a third-party tool for modelling software. It creates the possibility of taking modelling to the next level. Using VPL, designers can use a lot of scripting to create more complex models. This is usually not possible with the 
existing BIM tools. It also gives more flexibility to other actors in the project, by letting them set more parameters, in order to optimize the model even further.

\section{BUILDING INFORMATION MODELING (BIM)}

To help architects cope with the complexity of adaptive building requirements, processes and tools with abilities to generate the design of dynamic envelopes, which serve as shading devices, have become available. Nowadays, accurate information about all physical and functional features of a single building can be organised in a BIM.

$\mathrm{BIM}$ is one of the most important techniques in the architecture, engineering, construction, and operations (AECO) industry. A BIM is an emerging tool/methodology/ paradigm/process of virtual design and construction, involving the generation and management of digital representations of the physical and functional characteristics of a facility. It creates and uses the coordinated, consistent, computable information of the 3D models of the project's components, interconnected with the holistic information conceived as a source of shared knowledge. It aims to support decision-making. All of this allows for better analysis and control of a facility from its earliest conceptual stages, through design and construction, its operational life, and eventual demolition than manual processes. When accomplished, these computer-generated-semantic-3D models include accurate geometry and data required to support the construction, fabrication, and procurement activities through which the building is realized [4]-[9]. Today, a lot of tools exist to enable BIMs to evaluate global energy needs and increase the energy efficiency of buildings [10].

\section{PARAMETRIC DESIGN (PD)}

Parametric design is integrated within BIM to overcome the gap in research related to its inability to generate different forms. The Oxford Dictionary defines a parameter as "a numerical or other measurable factor contributing to a set that determines a system or adjusts the status of its operation," and the word parametric as "relating to or expressed in terms of a parameter or parameters." Based on the fundamentals of the literature, we can integrate PD in a design process, based on algorithmic thinking [11], which uses parameters and rules to constrain them [12], [13].

PD also relates to the BIM model as the latter uses PD's concepts of associative geometry and topological relationships [14], [15], which establish dependencies among different design elements.

\subsection{Parametric modelling and visual programming/scripting interfaces}

Parametric modelling and visual programming/scripting interface parametric modelling are also an important part of the BIM technology. They allow for the exploration of real-time design options and the management of the relationships among building elements. Parametric modelling makes parameters available for processing by computational algorithms and mathematical formulas, before being used or assessed.

Visual programming is a concept that provides designers with the means for constructing programmatic relationships utilizing graphical user interfaces, rather than writing codes from scratch. It allows building designers to easily experiment with various design alternatives, without professional knowledge of programming or scripting. There are various visual programming tools in BIM and CAD tools, such as Bentley Generative Components and Grasshopper for McNeel Rhinoceros. Architects are able to make different relationships by linking pre-packaged nodes together to make a custom algorithm $[10]$. 
The Autodesk Revit API (Application Programming Interface) helps designers add features to the software and create custom tools and plugins. Dynamo is a Revit plugin that utilizes the Revit API. It is built using the Windows Presentation Framework. As a parametric modelling engine, Dynamo is inspired by other tools to extend Revit's parametric modelling abilities, by adding an associativity, which is not available in the offthe-shelf application. This includes driving parameters based on external inputs, such as solar geometry. Dynamo helps users automate processes and computational design, through a node-based Visual Programming Interface [16].

\section{ORIGAMI (O) AS A FORM-FINDING TOOL}

Origami is the conventional Japanese craft of paper folding. The Japanese word "origami" is a combination of two Japanese words "ori" meaning to fold and "kami" meaning paper [17].

Origami, from an ancient Japanese craft to today's recently recognized "engineering discipline", offers many opportunities to engineers and designers to recognize mathematical relations, unprecedented forms, and static and dynamic structures [18].

\subsection{Reason to choose origami as an application for parametric modelling}

Origami has endless possibilities. However, the software and applications available for origami design are limited in terms of design freedom [18].

\subsection{Origami-based design}

In later years, building envelope explorations were carried out using inspiring new ideas from origami. Origami suggests a set of paper-folding methods, which can be tested with parametric modelling software. Origami is based on the relationships, as well as the shape, between different points and surfaces, which can be defined by rules. It can be turned into parametric models [19].

\section{BIMPO}

BimPo is a new approach integrating BIM, parametric design, and origami (see Fig. 1). This approach aims to overcome the previously mentioned gap, by generating various combinations that could improve daylight performance, as well as any other objective set by the designer. Hence, using this approach, a wide range of optimized solutions is reached.

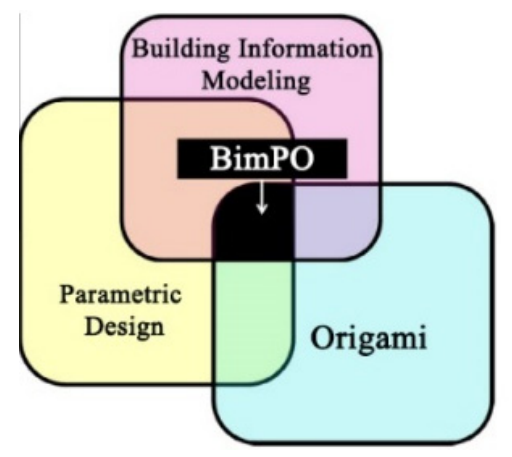

Figure 1: BimPO is a new approach to integrate BIM, parametric modelling, and origami. 


\section{METHODOLOGY}

The methodology used in this research is employed to identify, explain, and test a suggested, new approach. A research's aims and objectives are represented by input 1 (in1). By integrating existing related work (in2) with new knowledge gained through literature reviews and data collection (in3), the methodology can then generate the new approach (output or (out)), after passing through an iterative, three-stage theory-building process. This process includes three repetitive stages - description, scripting, and testing (see Fig. 2):

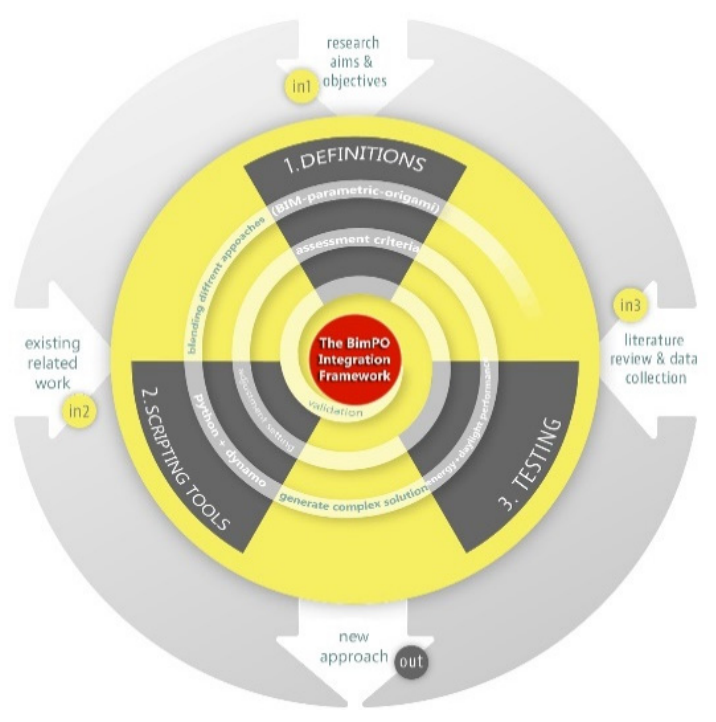

Figure 2: Methodology of the research.

- First, the Definition Stage identifies the benefits of blending the three different approaches: BIM, parametric modelling, and origami.

- Second, the Scripting Stage builds upon descriptions to infer the new theory, based on the integration of the three different approaches to generate complex dynamic components. In essence, the scripting stage develops a testable theoretical proposition, which clarifies what has previously been described.

- Third, the Testing Stage inspects the proposed script for validity, tests concepts or the relationships between them for accuracy, for energy efficiency (EE) and daylighting strategies, to develop the framework or theory.

\section{THEORETICAL PROCESS OF THE NEW APPROACH}

In this paper, the authors describe the design process of a set of OSDs developed using BimPO, and how this approach was integrated into the design workflow. The authors demonstrate how the integration of scripting in BIM influences the whole design process and also the selection of the final solution (see Fig. 3).

\section{CASE STUDY}

The goal of the project is to use the BimPO approach to generate alternatives for OSDs, based on the path of the sun in Alexandria, Egypt. For this project, the authors used: 


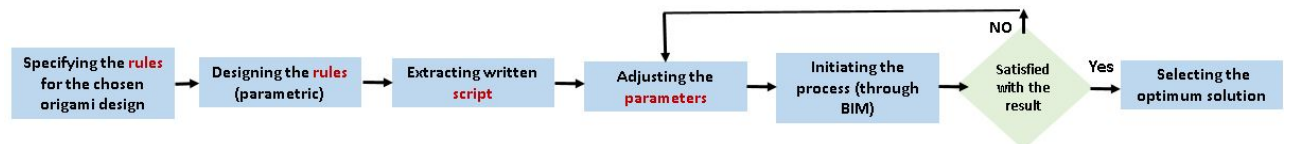

Figure 3: Theoretical process of the new approach.

- Revit architecture version 2021

- Dynamo version 2.1

- Refinery

\subsection{First stage}

In this stage, the authors identified the equation that controls the OSD (origamic model), by using the adaptive component in Revit 2021 (BIM) (see eqn (1) below):

$$
\text { base }=\operatorname{sqrt}\left(\left(\text { width factor }{ }^{2}\right)-\left({\text { opening } \left.\text { factor }^{2}\right)}\right)\right. \text {. }
$$

In this project, two OSD parameters are controlled in Dynamo.

1. Opening factor

2. Width factor (B_factor) (see Fig. 4).
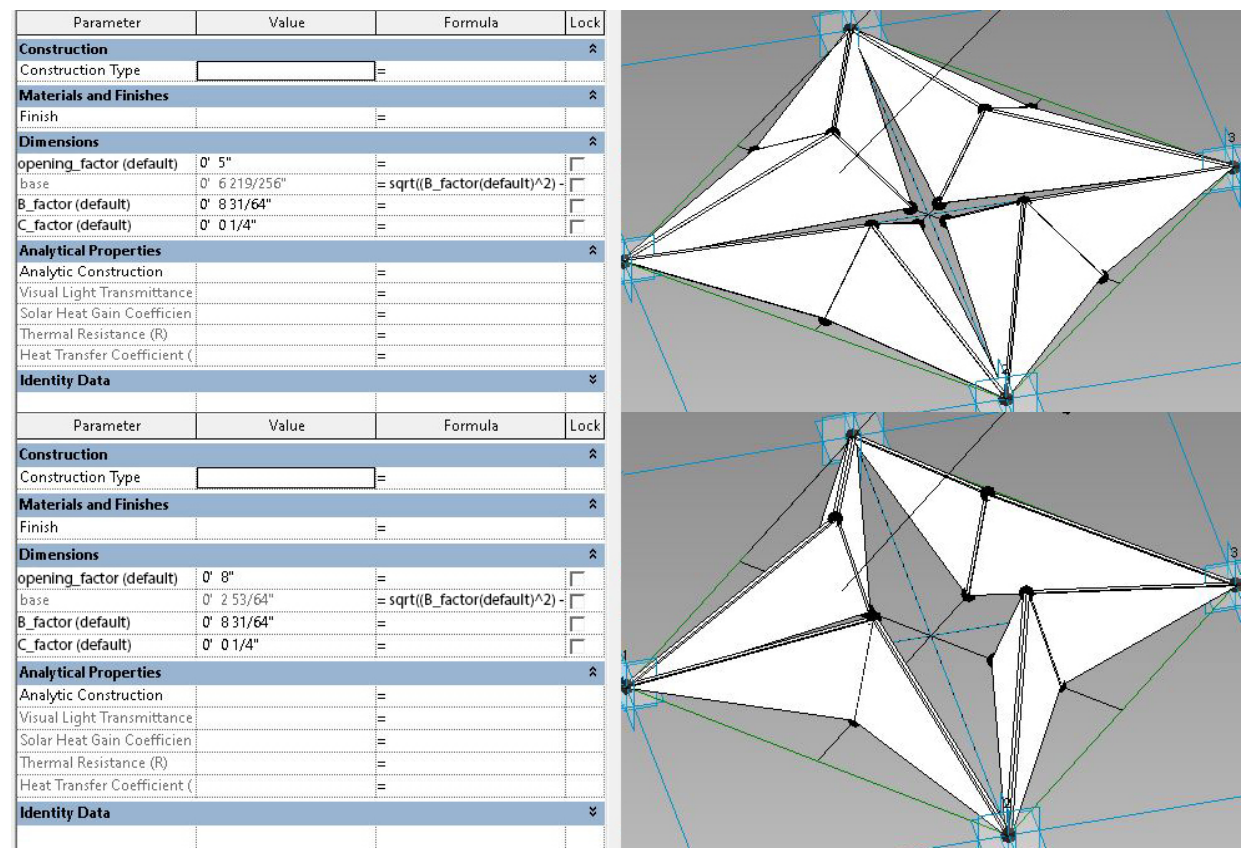

Figure 4: OSD parameters defined by the adaptive component. (Software: Revit 2021.)

A simple cylinder is created for the mass model and the adaptive components (OSD) are distributed on the surface of the cylinder (see Fig. 5). 


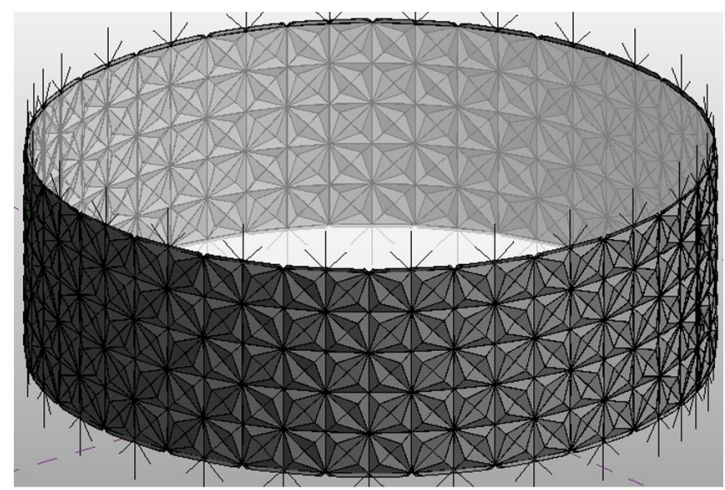

Figure 5: The adaptive components (OSD), surrounding the surface of a simple cylinder. (Software: Revit 2021.)

\subsection{Second stage (scripting)}

1. Exporting every single OSD coordinate, while utilizing exported data nodes in Dynamo.

2. Opening a new file and loading the adaptive component onto the file.

3. Importing the edited excel file using Dynamo (see Fig. 6).

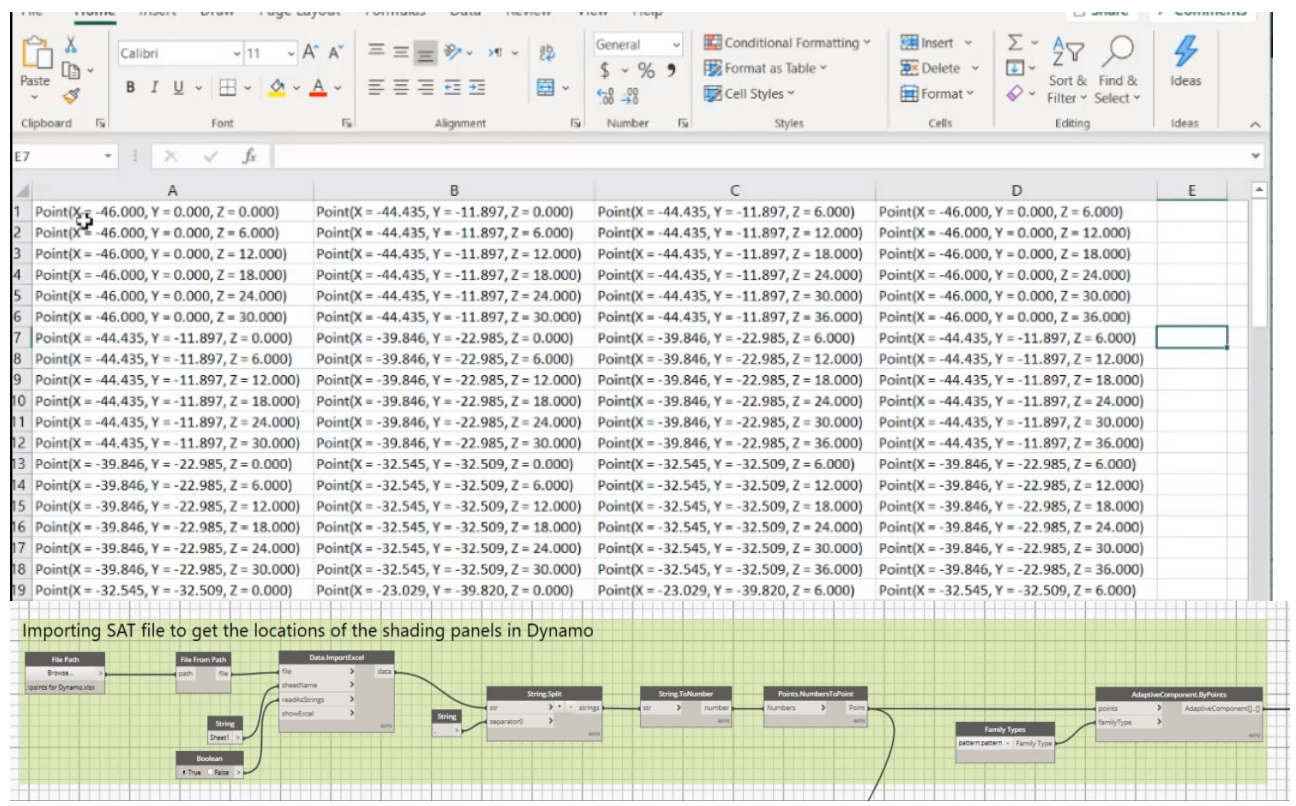

Figure 6: Graph showing steps from Dynamo for importing excel file to get the locations of origamic shading. (Software: Excel 2020 and Dynamo 2.1.)

4. Getting access to the parameters of the OSD by name (see Fig. 7). 


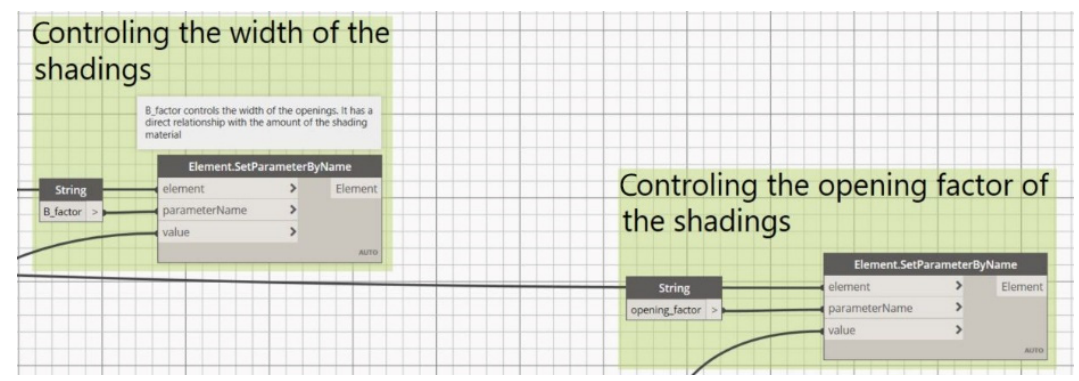

Figure 7: Graph showing steps from Dynamo to get access to the parameters of OSD by name. (Software: Dynamo 2.1.)

To reach the parametric values for the opening factor, the following procedure was followed:

1. Finding the normal values for each OSD (see Fig. 8).

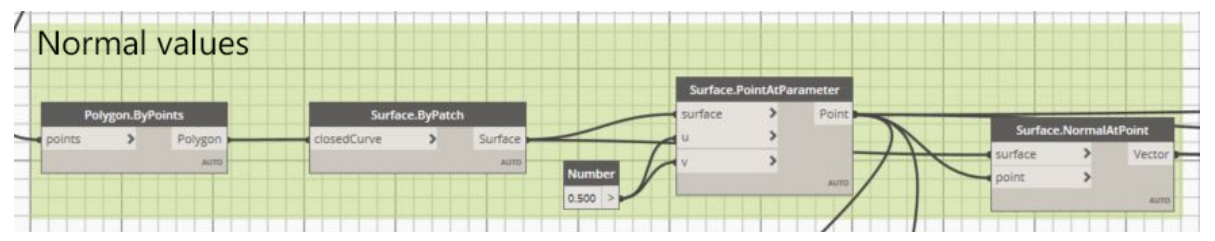

Figure 8: Graph showing steps from Dynamo to reach normal values for each OSD. (Software: Dynamo 2.1.)

2. Using three variables $(\mathrm{x}, \mathrm{y}, \mathrm{z})$ to create the position of the dynamic vector of the sun. In this procedure, the application of the sun's path in Revit was replaced by the vector of the sun present in Dynamo. This was done with information on the sun's position for Alexandria, Egypt in Revit. Those variables are used as input to generate alternatives with the help of Refinery optimization (see Fig. 9).

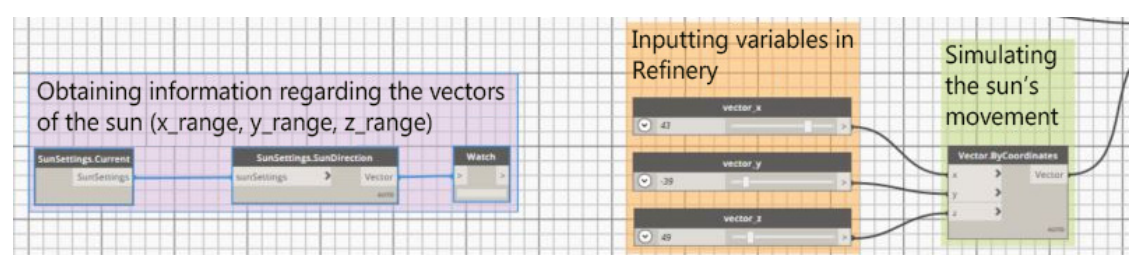

Figure 9: Graph showing steps from Dynamo for the input of three variables (x, y, z) into Refinery to generate the position of the dynamic vector of the sun. (Software: Dynamo 2.1.)

3. Calculating the angle between the normal values of OSD and the position of the sun's vector through the dot product and remapping the values of these angles for use as values for the opening factor (see Fig. 10). 


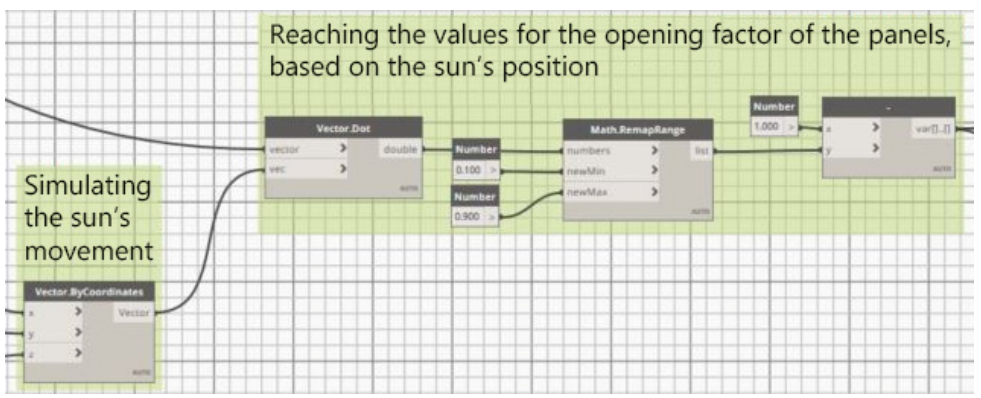

Figure 10: Graph showing steps from Dynamo to obtain the value of the opening factor of the OSD based on the sun's position. (Software: Dynamo 2.1.)

The same values were utilized to choose the colors of the OSD, based on the angle between the vector marking the sun's position and its normal values (see Fig. 11).

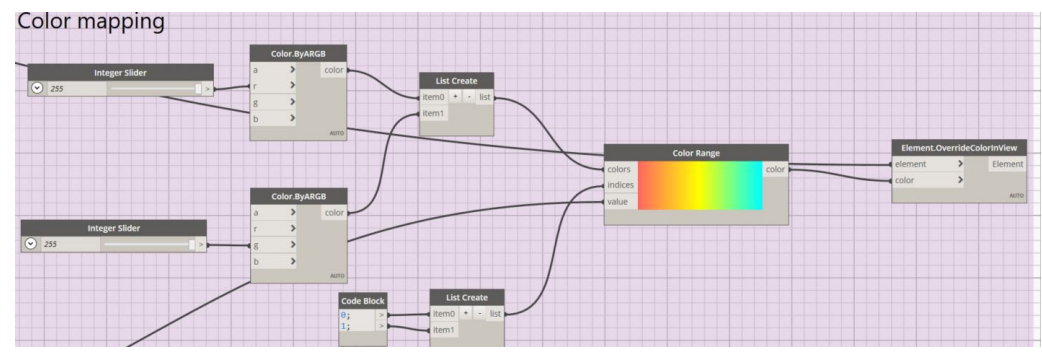

Figure 11: Graph showing steps from Dynamo to color the OSD. (Software: Dynamo 2.1.)

To obtain the parametric values for the width factor (B_factor), the following procedure is done:

1. Creating a mass model in Revit. This mass is the neighbouring building of the project (see Fig. 12).

Then, utilizing the boundary of the model as a reference to reach the distances between the closest points on the curve (building boundary) to the midpoints of the OSD. In this case, the boundary on the opposite side of the project was selected and was plotted on level 0 , as the reference curve (see Fig. 12).
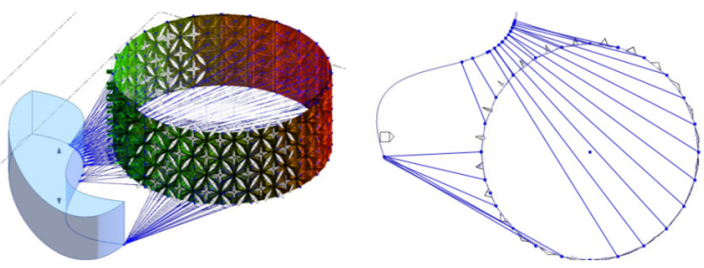

Figure 12: Obtaining the parametric values for the width factor utilizing the boundary of the model as a reference. (Software: Revit 2021.) 
2. Finding the distance between the midpoints of the OSD and the closest point to them on the chosen curve (see Fig. 13), then utilizing the remapped values to control the width factor of the OSD. This is done in order to get different values for the distance for later use in Refinery. The location on the curve changes through rotation. Three variables are used as input (see Fig. 14):

- $\mathrm{X}$ and $\mathrm{y}$ : to change the origin of the rotation

- Angle: to change the angle of the rotation

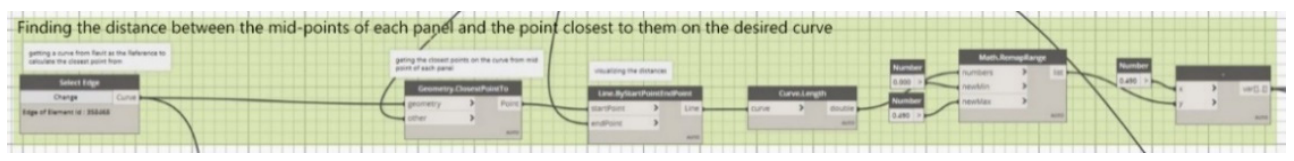

Figure 13: Graph from Dynamo showing steps to find the distance between OSD points and the curve. (Software: Dynamo 2.1.)

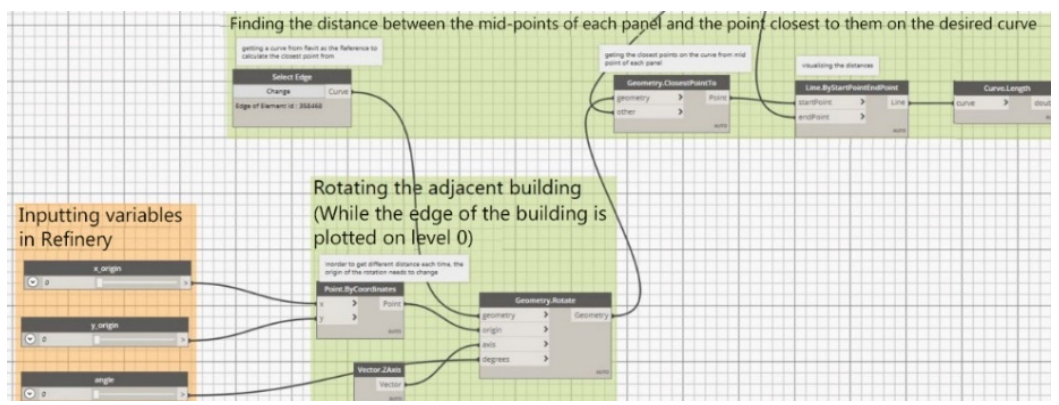

Figure 14: Graph explaining the use of the three variables as input to change the angle of rotation. (Software: Dynamo 2.1.)

\subsection{Third stage}

The script is exported to Refinery. There are two objectives for the use of Refinery to generate different alternatives (see Fig. 15). They are:

1. Maximizing the value of the summation of the values of the opening factor

2. Minimizing the value of the summation of the values of the width factor

The generation process is created with a population size of 48 and leads to 20 alternatives.
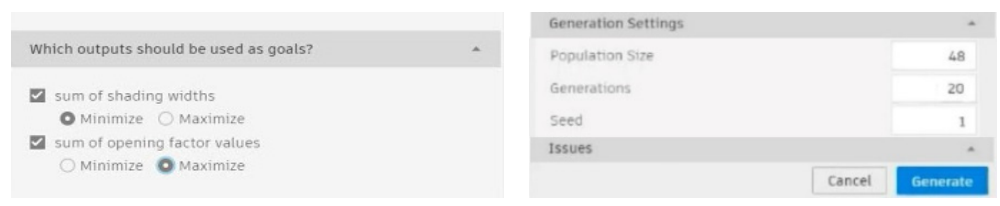

Figure 15: Customization options for the generation of alternative solutions. (Software: Refinery.) 


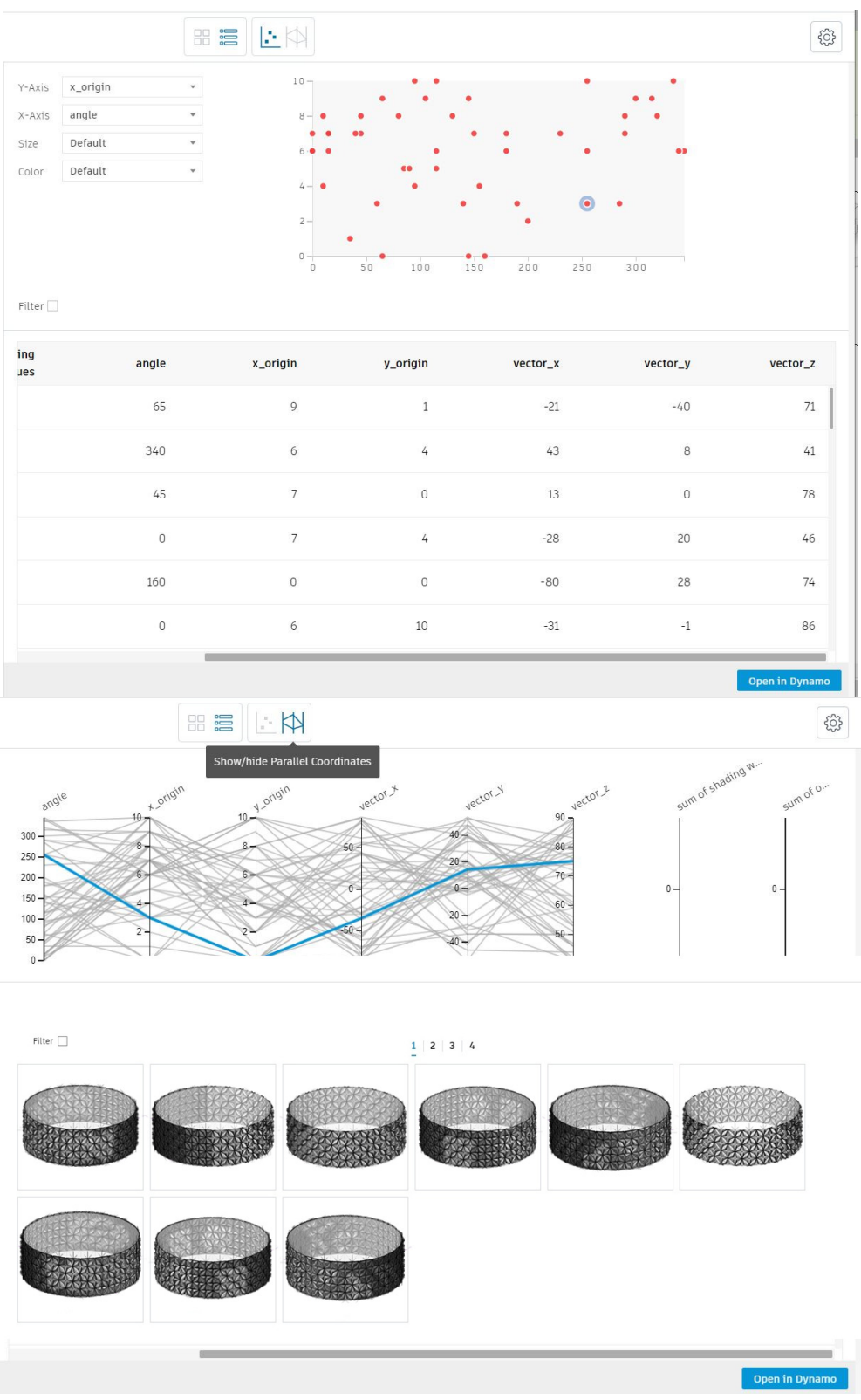

Figure 16: Generating alternative solutions. (Software: Refinery.)

These alternatives show the solutions in 20 alternative generations (see Fig. 16), according to the determined objectives. Any alternative solution could be selected to be displayed as a Revit project and its corresponding variables could be extracted. One solution is selected in Refinery (see Fig. 17). Architects can test their solutions based on many concepts (energy efficiency, daylight strategy, etc.). 


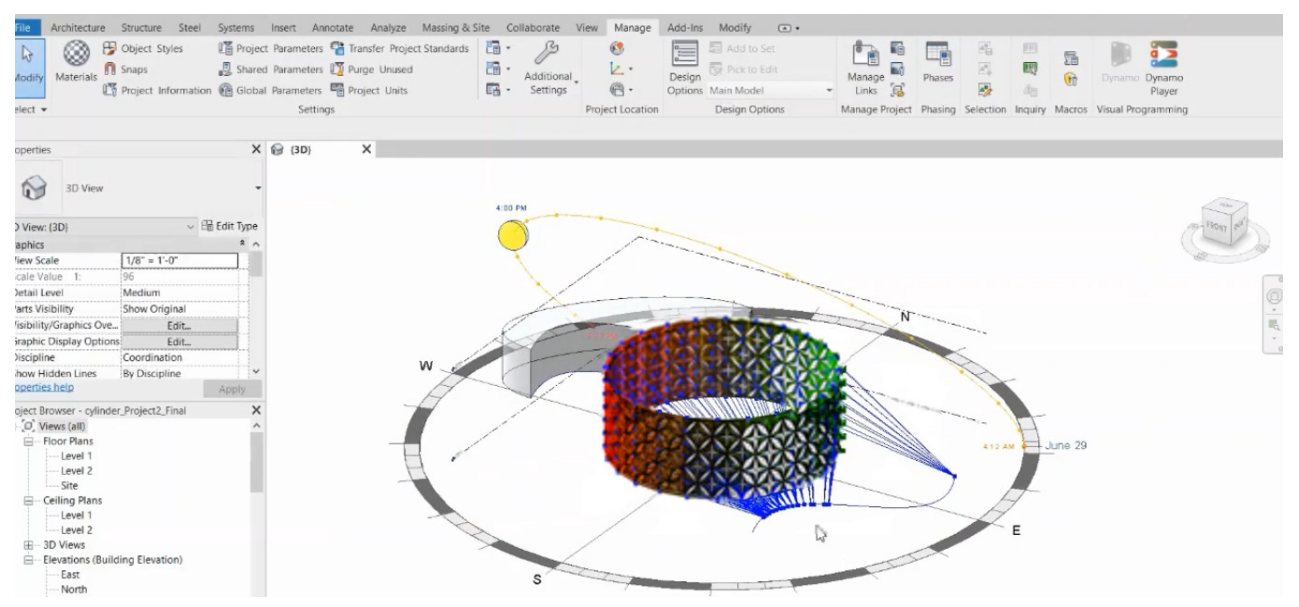

Figure 17: The final selected solution. (Software: Revit 2021.)

\section{CONCLUSIONS}

It is very important to provide more opportunities for form generation. Currently, there is gap between new trends and the different types of software that architects use. Basically, there is an absence of basic programming knowledge, which in turn makes an architect implement his/her creative ideas no matter the difficulties.

This is the purpose for the use of BimPO, a new method used to develop the BIM platform, using VPL and its capacity to integrate BIM, parametric design, and origami.

This exploration was accomplished in order to establish early design processes, which are effective as alternative solutions to isolate design problems associated with complex BIM façades design, responding to environmental changes.

Nowadays, the potential for the use of technology is impressive. Studies to be pursued in this field should now put more focus on this method. It is important to understand that new technologies require conducting new research and applying new frameworks. The legislative, professional, and economic apparatus is currently not prepared to work with this philosophy. However, it is absolutely necessary to catch up with the times.

The study regarding the method performed in this research could also be applied in other areas of design. It represents a line of thought to share with different disciplines, to facilitate the integration of technology.

\section{ACKNOWLEDGEMENTS}

The authors would like to acknowledge the effort made by Professor Ali F. Bakr, as well as all members of the research team to make the case study possible.

\section{REFERENCES}

[1] Malleson, A.K., NBS International BIM Report 2018, NBS: United Kingdom, 2018.

[2] Malleson, P., Kato, H., Popíšilová, B., Watson, D. \& Friborg, G., NBS International BIM Report 2016, NBS: United Kingdom, 2018.

[3] Finances, Best 20 Building Information Modeling (BIM) Software in 2018, 2018. https://financesonline.com/building-information-modeling/.

[4] Krygiel, E. \& Nies, B., Green BIM: Successful Sustainable Design with Building Information Modeling, John Wiley \& Sons: New Jersey, 2008. 
[5] Kymmell, W., Building Information Modeling: Planning and Managing Construction Projects with $4 D$ CAD and Simulations, McGraw-Hill: New York, 2008.

[6] Eastman, C., Teicholz, P., Sacks, R. \& Sacks, K., BIM Handbook - A Guide to Building Information Modeling for Owners, Managers, Designers, Engineers, and Contractors. John Wiley \& Sons, Inc.: New Jersey, 2011.

[7] Matthew, G., Jason, G., Melissa, T., Seokho, C. \& Fiona, C., Building information modelling: An international survey. World Building Congress, 2013, pp. 5-9 May 2013, Brisbane, Australia, 2013.

[8] Ladenhauf, D. et al., Computational geometry in the context of building information. Energy and Buildings, 115, pp. 78-84, 2015.

[9] Agugiaro, G., Energy planning tools and city GML-based 3D virtual city models: Experiences from Tento (Italy). Applied Geomatics, 8(1), pp. 41-56, 2016.

[10] Kim, H. \& Yan, W., Parametric BIM-based energy simulation for buildings with complex kinetic facades. Proceedings of the 33rd eCAADe Conference, Vienna, Austria, 2015.

[11] Jabi, W., Soe, S., Theobald, P., Aish, R. \& Lannon, S., Enhancing parametric design through non-manifold topology. Design Studies, pp. 1-19, 2017.

[12] Barrios, C., Parametric affordances: What, when, how. Proceedings of the ACADIA Regional Conference, 2011.

[13] Gero, J., An empirical foundation for design patterns in parametric design. Proceedings of the 20th International Conference of the Association for ComputerAided Architectural Design Research in Asia, 2015.

[14] Gerber, D. \& Pantazis, E., A multi-agent system for facade design: A design methodology for design exploration, analysis and simulated robotic fabrication. Proceedings of the 36th Annual Conference of the Association for Computer Aided Design in Architecture, 2016.

[15] Oxman, R., Thinking difference: Theories and models of parametric design thinking. Design Studies, 2017.

[16] Kensek, K.M., Integration of environmental sensors with BIM: Case studies using Arduino, Dynamo, and the Revit API. Informes de la Construcción, 66(536), pp. 3139, 2014. DOI: 10.3989/ic.13.151.

[17] Gavy, Origami Inspired Architecture and Interior Design - What Creativity Does, 2012.

[18] Tantawy, D., Origamic architectural form design system. International Journal of Sciences: Basic and Applied Research, 21(2), pp. 66-86, 2015.

[19] Gao, W. \& Ramani, K., Kaleidogami тм: Multi-Primitive Reconfigurable Artistic Structures. School of Mechanical Engineering School, Electrical and Computer Engineering, Purdue University. 